\title{
Demo: TinySDR, A Software-Defined Radio Platform for Internet of Things
}

\author{
Mehrdad Hessar \\ University of Washington \\ Seattle, Washington \\ mehrdadh@uw.edu \\ Vikram Iyer \\ University of Washington \\ Seattle, Washington \\ vsiyer@uw.edu
}

\author{
Ali Najafi \\ University of Washington \\ Seattle, Washington \\ anajafi@uw.edu \\ Shyamnath Gollakota \\ University of Washington \\ Seattle, Washington \\ gshyam@uw.edu
}

\begin{abstract}
Wireless protocol design for IoT networks is an active area of research. We demonstrate tinySDR which is a low-power software-defined radio platform tailored to the needs of IoT endpoints. TinySDR is a standalone, fully programmable software-defined radio platform which has the requirements of IoT protocols. We present the physical layer implementations of BLE beacon and LoRa protocols to demonstrate the capabilities of tinySDR.
\end{abstract}

\section{CCS CONCEPTS}

- Hardware $\rightarrow$ Networking hardware; Wireless devices.

\section{KEYWORDS}

IoT, Radio platform, Software-defined radio, low-power

\section{ACM Reference Format:}

Mehrdad Hessar, Ali Najafi, Vikram Iyer, and Shyamnath Gollakota. 2019. Demo: TinySDR, A Software-Defined Radio Platform for Internet of Things. In The 25th Annual International Conference on Mobile Computing and Networking (MobiCom '19), October 2125, 2019, Los Cabos, Mexico. ACM, New York, NY, USA, 3 pages. https://doi.org/10.1145/3300061.3343373

\section{INTRODUCTION}

Recent years have seen development of numerous wireless protocols for Internet of Things (IoT) devices. New protocols including LoRa, Sigfox and NB-IoT have been developed

Permission to make digital or hard copies of part or all of this work for personal or classroom use is granted without fee provided that copies are not made or distributed for profit or commercial advantage and that copies bear this notice and the full citation on the first page. Copyrights for thirdparty components of this work must be honored. For all other uses, contact the owner/author(s).

MobiCom '19, October 21-25, 2019, Los Cabos, Mexico

(c) 2019 Copyright held by the owner/author(s).

ACM ISBN 978-1-4503-6169-9/19/10.

https://doi.org/10.1145/3300061.3343373

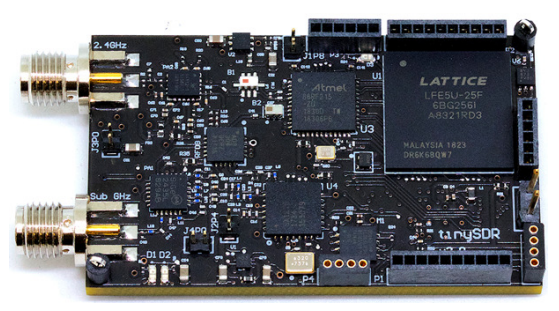

Figure 1: TinySDR Hardware

to extend the range of wireless communication for IoT devices. These protocols require dedicated radio chipset which provides little or no flexibility for academic community to innovate on them [4], let alone developing new protocols from the ground up.

Ideally, We would like a software-defined radio (SDR) platform which provides flexibility for implementing different IoT protocols and also be standalone. Further, since many IoT protocols are designed for deployment at large scale, these endpoints need to be low power to run on batteries which facilitate the deployment without dedicated power access and in mobile scenarios. In [5], we presented tinySDR, the first SDR platform that satisfies these requirements.

In this demonstration, we showcase the functionality of tinySDR, by implementing the physical layer of two common IoT protocols in two different ISM frequency bands: i) LoRa modulator which is a $900 \mathrm{MHz}$ IoT protocol targeted for long-range applications and ii) Bluetooth Low Energy (BLE) beacon which is a common $2.4 \mathrm{GHz}$ IoT protocol. In the rest of this demonstration, we briefly describe the hardware design of tinySDR and then our physical layer implementations on tinySDR platform.

\section{HARDWARE DESIGN}

We seek to minimize power consumption and cost while offering the flexibility of an SDR to process raw samples. The core of our platform is the programmable and flexible 


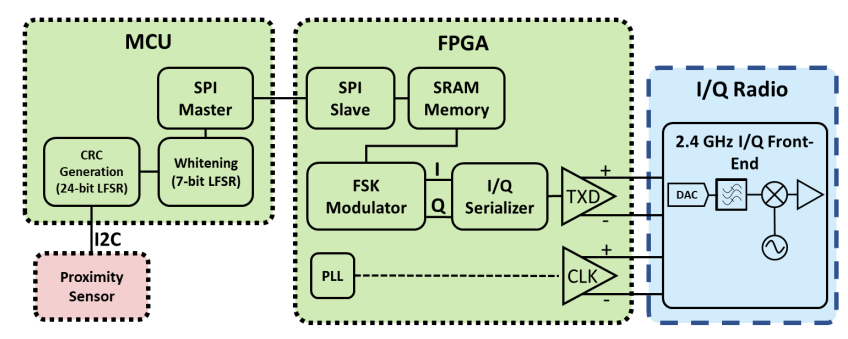

Figure 2: BLE Beacon Implementation on TinySDR.

PHY layer that processes and converts bits to radio signals and vice versa. To have flexible PHY layer, we need to have flexible radio front-end supporting sending and receiving raw I/Q samples and flexible I/Q data processing module. We use AT86RF215 [1] which integrates LNA, programmable receiver gain, ADC, DAC and low-pass filter. This radio supports both $900 \mathrm{MHz}$ and $2.4 \mathrm{GHz}$ frequency bands and has $4 \mathrm{MHz}$ bandwidth. We interface radio chip with a low-power FPGA from Lattice Semiconductor, LFE5U-25F [3], for baseband processing operations, physical layer implementation and sending/receiving I/Q samples to/from radio.

TinySDR uses a Cortex M4F microcontroller, MSP432 [2], as central controller. We interface MCU with FPGA using SPI interface to exchange data during both TX and RX operations. We use MCU to control radio operations, amplifiers and RF switches. The MSP432 MCU on tinySDR has $64 \mathrm{~KB}$ of onboard SRAM memory and $256 \mathrm{~KB}$ on-board flash memory and consumes less than $1 \mathrm{uA}$ during sleep operation which is great choice for a low-power IoT platform. Finally, we use MCU to control power supply for each unit in tinySDR to optimize power consumption during different operations.

While the above discussion enables a small and low power SDR for easy deployment, FPGAs and microcontrollers typically require a wired interface for reprogramming. TinySDR is designed to use over-the-air (OTA) update system to reconfigure and program itself wirelessly. To enable this, we use a LoRa radio that has long-range coverage and supports different data rates. This allows us to trade off rate for range depending on the deployment scenario. Further details about OTA are discussed in [5].

\section{DEMO DESCRIPTION}

Here, we demonstrate two case studies using tinySDR. First we explain our BLE beacon implementation and then we demonstrate our LoRa modulator implementation on tinySDR.

\subsection{BLE Beacons with TinySDR}

To demonstrate tinySDR's $2.4 \mathrm{GHz}$ capabilities we implement BLE beacons which are commonly used by IoT devices. We implement non-connectable BLE advertisements which

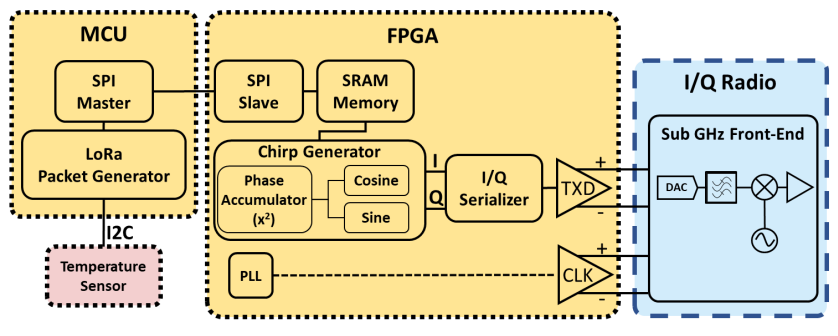

Figure 3: LoRa Modulator Implementation on TinySDR.

are broadcast packets used for beacons. These packets allow a low power device to broadcast its data to any listening receiver within range without the power overhead of exchanging packets to setup a connection.

Bluetooth advertisements consist of 6-37 octets, beginning with fixed preamble and access address fields indicating the packet type set to 0xAA and 0x8E89BED6 respectively. This is followed by the packet data unit (PDU) beginning with a 2 byte length field and followed by a manufacturer specific advertisement address and data. The final 3 bytes of the packet consist of a CRC generated using a 24-bit linear feedback shift register (LFSR) with the polynomial $x^{24}+x^{10}+x^{9}+x^{6}+x^{4}+x^{3}+x+1$. The LFSR is set to a starting state of $0 \times 555555$ and the PDU is input LSB first. The final LFSR state after inputting the PDU becomes the CRC. Data whitening is then performed over the PDU and CRC fields to eliminate long strings of zeros or ones within a packet. This is also done using a 7-bit LFSR with polynomial $x^{7}+x^{4}+1$. The LFSR is initialized with the lower 7 bits of the channel number the packet will be transmitted on, and each byte is input LSB first.

To transfer the bitstream of BLE beacon to FPGA for transmission operation we use SPI interface and write BLE packet in FPGA's internal SRAM. Then we read the bitsream from SRAM memory and feed that to the FSK modulator. FSK modulator generates I/Q samples based on the input bitstream which are passed to I/Q serializer. Finally, serialized I/Q samples sent to the I/Q radio using low-voltage differential signaling (LVDS) interface between FPGA and I/Q radio. We feed I/Q samples to $2.4 \mathrm{GHz}$ front-end of I/Q radio to generate BLE packets at $2.4 \mathrm{GHz}$ ISM band. We show that we can receive these BLE beacons on an iPhone 6s.

\subsection{LoRa Modulator with TinySDR}

We choose LoRa as it is gaining popularity for IoT solutions due to its long range capabilities. We describe the basics of LoRa modulation followed by the implementation of our the modulator. 
LoRa achieves long ranges by using Chirp Spread Spectrum (CSS) modulation. In CSS, data is modulated using linearly increasing frequency upchirp symbol $[4,8]$. Each upchirp symbol has two main features: Spreading Factor (SF) and Bandwidth (BW). SF determines the number of bits in each upchirp symbol and BW is the difference between upper and lower frequency of the chirp which together with SF determines the length of an upchirp symbol. SF and BW trade data rate for range. Data is modulated by $2^{S F}$ cyclicshifts of an upchirp symbol. The starting point of the symbol in frequency domain, which is the cyclic shift of the upchirp symbol, determines its value.

Fig. 3 shows the block diagram of our LoRa modulator implementation on tinySDR. We use MCU to generate LoRa packet and stream the packet to FPGA using SPI interface. First, we store the LoRa symbol values in FPGA's SRAM memory. Then, we read them from SRAM and feed them to the chirp generator module which generates the I/Q samples using a squared phase accumulator and two lookup tables for $\operatorname{Sin}$ and $\operatorname{Cos}$ function. We then feed these I/Q samples into I/Q Serializer to stream them over the LVDS interface to the I/Q radio. The radio upconverts these samples and transmit the data to the antenna. We show that we can receive LoRa packets using a Semtech SX1276 LoRa chipset without any sensitivity degradation.

\section{CONCLUSION}

This demonstration presents two protocol implementations on tinySDR. TinySDR can be used for IoT research such as designing PHY/MAC protocols, research on IoT localization, machine learning on IoT devices and custom backscatter reader for backscatter systems [6, 7]. Please refer to [5] for full details of tinySDR platform.

\section{ACKNOWLEDGEMENT}

This work was funded in part by NSF awards CNS-1812554, CNS-1452494, CNS-1823148 and Google Faculty Research Awards.

\section{REFERENCES}

[1] 2016. AT86RF215. http://ww1.microchip.com/downloads/en/devicedoc/ atmel-42415-wireless-at86rf215_datasheet.pdf.

[2] 2017. MSP432P401M SimpleLink Mixed-Signal Microcontrollers Datasheet. http://www.ti.com/lit/ds/symlink/msp432p401r.pdf.

[3] 2018. LFE5U FPGA Family Datasheet. http://www.latticesemi.com/ view_document?document_id=50461.

[4] M. Hessar, A. Najafi, and S. Gollakota. 2019. NetScatter: Enabling Large-Scale Backscatter Networks. In 16th \{USENIX\} Symposium on Networked Systems Design and Implementation (\{NSDI $\}$ 19).

[5] Mehrdad Hessar, Ali Najafi, Vikram Iyer, and Shyamnath Gollakota. 2019. TinySDR: Low-Power SDR Platform for Over-the-Air Programmable IoT Testbeds. arXiv preprint arXiv:1907.02063 (2019).

[6] Saman Naderiparizi, Mehrdad Hessar, Vamsi Talla, Shyamnath Gollakota, and Joshua R Smith. 2018. Towards battery-free HD video streaming. In 15th USENIX Symposium on Networked Systems Design and Implementation (NSDI 18).

[7] Ali Saffari, Mehrdad Hessar, Saman Naderiparizi, and Joshua R Smith. 2019. Battery-Free Wireless Video Streaming Camera System. In 2019 IEEE International Conference on RFID (RFID). IEEE, 1-8.

[8] V. Talla, M. Hessar, B. Kellogg, A. Najafi, J. R. Smith, and S. Gollakota. 2017. LoRa Backscatter: Enabling The Vision of Ubiquitous Connectivity. Proceedings of the ACM on Interactive, Mobile, Wearable and Ubiquitous Technologies (2017). 